\title{
A RISING THREAT - RISK FACTORS AND OUTCOMES RELATED TO INFECTIONS WITH ACINETOBACTER SPECIES
}

\author{
PRERNA KHURANA ${ }^{1}$, SHRIKALA BALIGA ${ }^{2 *}$, SUCHITRA SHENOY M $^{2}$, PRASANNA MITHRA ${ }^{3}$
}

${ }^{1}$ Department of Infectious Diseases, Division of Internal Medicine, Medanta - The Medicity, Gurgaon, India. ${ }^{2}$ Department of Microbiology, KMC, Mangalore, Karnataka, India. ${ }^{3}$ Department of Community Medicine, KMC, Mangalore, Karnataka, India. Email: shrikala@yahoo.com

Received: 26 September 2016, Revised and Accepted: 09 December 2016

\section{ABSTRACT}

Objective: Acinetobacter species is an important cause of community as well as nosocomial infections with a high mortality rate. The study was done to analyze the risk factors associated with Acinetobacter infections and their outcomes.

Methods: The clinical details of 100 patients having infections with Acinetobacter species over a period of 1-year were analyzed for underlying risk factors and outcomes. The antibiotic sensitivity results were interpreted according to the Clinical Laboratory Standards Institute guidelines.

Results: Majority of the infections caused by the Acinetobacter species were lower respiratory tract infections, most common being ventilatorassociated pneumonia. $47 \%$ of the isolates were multi drug resistant and $26 \%$ were extensively drug resistant. There is a significant chance of drug resistance and a poor outcome with intensive care unit (ICU) stay, prolonged hospital stay of more than 7 days, the presence of 5 or more risk factors. Endotracheal intubation and mechanical ventilation were the risk factors for increased drug resistance in the ICU. Drug resistance was also seen more frequently in patients with chronic obstructive pulmonary disease, chronic kidney disease, and patients on post-operative care.

Conclusion: The steady increase in drug resistant Acinetobacter species and limited antibiotics available advocates an uncompromising approach toward infection control and a judicious use of antibiotics especially in the ICU. An understanding about the risk factors helps in the appropriate approach and management of the patient.

Keywords: Acinetobacter, Risk factors, Invasive procedures, Nosocomial.

(C) 2017 The Authors. Published by Innovare Academic Sciences Pvt Ltd. This is an open access article under the CC BY license (http://creativecommons. org/licenses/by/4. 0/) DOI: http://dx.doi.org/10.22159/ajpcr.2017.v10i3.15364

\section{INTRODUCTION}

Acinetobacter baumannii has been notified by the Infectious Disease Society of America as "red alert" pathogen [1]. They are opportunistic pathogens and cause both community acquired and health-care associated infections such as nosocomial pneumonia, meningitis, urinary tract infection, skin and soft tissue infection and bacteremia, with a high mortality rate [2]. With the acquisition of antibiotic resistance genes, there is an increase in the prevalence of multi-drug resistance (MDR) in Acinetobacter species [3].

This study was done to analyze the factors responsible for infections caused by Acinetobacter species and those associated with infection due to MDR Acinetobacter strains.

\section{METHODS}

This study is a prospective study conducted in intensive care units (ICUs) and wards of a tertiary care hospital in Mangalore, Karnataka, over a period of 1-year. 100 patients with infections caused by Acinetobacter species were analyzed for associated risk factors using the medical records of each patient. All outpatients and patients with colonization with Acinetobacter species were excluded from the study. Non-random sampling method was adopted for the study. The following variables were analyzed: ICU admission, duration of stay in the hospital, mechanical ventilation, endotracheal intubation, the presence of underlying diseases or conditions, including chronic obstructive pulmonary disease (COPD), diabetes mellitus, hypertension, chronic kidney disease (CKD), chronic liver disease, ischemic heart disease, malignancy, past history of device implantation or surgery, and instrumentation at various sites such as Central venous catheters [4-7]. Ventilator-associated pneumonia (VAP) is defined according to the American Thoracic Society as pneumonia that occurs 48-72 hrs or thereafter following endotracheal intubation, characterized by the presence of a new or progressive infiltrate, signs of systemic infection (fever, altered white blood cell count), changes in sputum characteristics, and detection of a causative agent [8].

The antibiotic susceptibility test results of these isolates of Acinetobacter species were interpreted according to Clinical Laboratory Standards Institute recommendations [9-11]. MDR Acinetobacter isolates were defined as non-susceptible to one or more agent in three or more antimicrobial categories. Extensively drug resistant (XDR) isolates were defined as non-susceptible to one or more agents in all but two or less antimicrobial categories, and pan-drug resistant (PDR) strains were defined as non-susceptible to all antimicrobial agents [12]

\section{RESULTS}

Out of the total 100 patients included in the study, 63 were males and 37 were females. Maximum isolates (46 out of 100) were from the elderly population, i.e., $>55$ years of age, followed by 23 isolates in the age group 36-55 years, 16 isolates in the age group 16-35 years, 13 isolates in the neonates and 2 in children till 15 years of age.

The majority of the infections caused by Acinetobacter species occurred in patients admitted in the ICUs (73\%). 61\% of infections in our study were lower respiratory tract infections (LRTIs), out of which $54.1 \%$ (33 out of 61) were VAP. The specimen used were endotracheal tube secretions (35), sputum (21), and broncho alveolar lavage (5) (Table 1). To diagnose LRTI or VAP, strict diagnostic criteria was used and all colonizers were excluded from the study. 
All the skin and soft tissue infections were post-operative cases where Acinetobacter species were isolated from the pus and wound swab specimens collected from the operated site.

About $72 \%$ of the infections caused by Acinetobacter species in the study were monomicrobial, while $28 \%$ were polymicrobial, most commonly associated with Klebsiella species (14\%), followed by Pseudomonas species (5\%), Enterococcus species (3\%), Staphylococcus aureus (3\%), Citrobacter species (2\%), and Candida species (2\%).

Risk factors associated with these infections caused by Acinetobacter species were analyzed. The most common risk factor for the development of infection with Acinetobacter species was intubation followed by mechanical ventilation and insertion of medical devices (Table 2). Diabetes was associated with 29 out of 100 patients.

Around $47 \%$ of these isolates were MDR while $26 \%$ of these isolates were XDR. There were no PDR strains.

$67 \%$ of the patients suffering from the infections caused by Acinetobacter species improved, while $22 \%$ of the patients expired. $7 \%$ of the patients worsened while in $4 \%$, the condition remained unchanged.

The majority of patients, i.e., $83 \%$ had a prolonged duration of hospital stay (7 days or more). Patients with prolonged ICU stay, i.e., more than 7 days had increased association with infections caused by MDR Acinetobacter species and also a poor outcome, i.e., worsening of the condition or mortality $(\mathrm{p}<0.05)$. There was significant chance of the Acinetobacter species being drug resistant when five or more risk factors were present $(\mathrm{p}<0.05)$. Mortality increased significantly when it was associated with one or more risk factors $(p<0.001)$. When each of the risk factors was analyzed to risk of mortality and drug resistance, it was observed that only mechanical ventilation was significantly associated with both of them $(\mathrm{p}<0.05)$. But in the ICU settings, other risk factors such as endotracheal intubation, mechanical ventilation, post-operative case, COPD and CKD were also significantly associated with drug resistance $(\mathrm{p}<0.05)$.

Table 1: Specimen wise isolation of Acinetobacter species

\begin{tabular}{ll}
\hline Specimen & Number $(\mathbf{n = 1 0 0})$ \\
\hline ET secretions & 27 \\
Sputum & 21 \\
Pus and wound swab & 14 \\
Blood & 10 \\
Oral suction catheter & 08 \\
CSF & 5 \\
Urine & 5 \\
BAL & 5 \\
Umbilical catheter tip & 3 \\
Central line tip & 2 \\
\hline
\end{tabular}

ET: Endotracheal tube, CSF: Cerebral spinal fluid, BAL: Broncho alveolar lavage

Table 2: Risk factors associated with infection due to Acinetobacter species

\begin{tabular}{ll}
\hline Risk factor & Number \\
\hline Intubation & 81 \\
Mechanical ventilation & 36 \\
Instrumentation & 35 \\
Diabetes & 29 \\
Hypertension & 26 \\
Post-operative case & 25 \\
COPD & 19 \\
Ischemic heart disease & 12 \\
CKD & 13 \\
Chronic liver disease & 5 \\
Malignancy & 7 \\
\hline
\end{tabular}

COPD: Chronic obstructive pulmonary disease, CKD: Chronic kidney disease

\section{DISCUSSION}

Acinetobacter species, once considered to be a part of the commensal flora of humans, now pose a serious challenge, especially in healthcare settings where it is now an important cause of life-threatening infections. The ability to survive for prolonged duration in the hospital settings and the rapid acquisition of MDR has made infections due to Acinetobacter species, a major concern in health-care settings.

We found increased incidence of infection in males (63\%) and a male:female ratio of 1.7:1. LRTI due to Acinetobacter species was the most common infection in our study (61\%). Most patients $(63.9 \%)$ with LRTI belonged to the $>55$ years age group. $65.6 \%$ males had LRTI compared to only $34.4 \%$ of females which could be related to the increased history of smoking among men. Smoking damages the cilia in the respiratory tract and alters the structural and immune defenses of the host predisposing the host to infections. Advanced age is a risk factor for acquiring infection with Acinetobacter species which could be attributed to the poor immune defense mechanisms in the elderly age group and the presence of coexisting comorbidities and chronic diseases. Other studies on infections with Acinetobacter species also reported male preponderance and increased incidence in older group $[6,13]$.

Nearly $35(57.4 \%)$ of the isolates from LRTI were isolated from endotracheal tips and aspirates, $21(34.4 \%)$ from sputum and $5(8.2 \%)$ from bronchoalveolar lavage. $54.1 \%$ of these patients with LRTI had developed VAP. $14 \%$ of the infections were skin and soft tissue infections while $10 \%$ of the infections were blood stream infections. Similar results were obtained in the study done by Prashanth and Badrinath, in which they found respiratory tract infections to be the most common infection caused by Acinetobacter species (51.2\%), followed by bloodstream infections (16.2\%) [5]. In another study done by Lee et al., respiratory infections were the most common infections caused by both imipenemresistant and imipenem-sensitive A. baumannii isolates, 57\% and $64 \%$, respectively [14]. The type of infections caused depends on the condition of the patients and the type of interventions and procedures performed, and thus varies from hospital to hospital. In our setup, 81\% of the patients had endotracheal intubation and $36 \%$ of the patients were mechanically ventilated.

Mixed infections, combining Acinetobacter species along with other organisms have been found to be more virulent. These infections are more resistant to treatment and mortality is high in these patients like in studies by Telley and Roberts [15]. 28\% of our patients had mixed infections. Most of these polymicrobial infections were from respiratory tract specimens $(78.6 \%)$ and Klebsiella pneumoniae was the most common associated organism isolated from polymicrobial infections, followed by Pseudomonas species. In our study, $57.1 \%$ of the mixed infections were due to XDR Acinetobacter strains, $28.6 \%$ were due to MDR Acinetobacter strains. 53.6\% of the mixed infections in our study were associated with mortality and poor outcome.

We found a strong association between ICU admission and development of infection with Acinetobacter species $(\mathrm{p}<0.05)$. The majority of the patients included in the study were admitted to the ICU $(73 \%)$, which indicates that ICU admission is a predisposing factor for the occurrence of infections caused by Acinetobacter species. Similar results were found by Tripathi et al. [16], Lone et al. [6] who found that Acinetobacter infections in the ICU were significantly more common than those in the general ward. Patients in ICU are sicker and require more invasive monitoring and therapeutic procedures. The environmental contamination of the ICU and transmission of the infection from colonized patients is probably another important source of infection due to Acinetobacter species. The development of ICU-acquired infections is associated with worse outcomes including increased morbidity and mortality [17]. 75.3\% (55 out of 73) of the patients admitted to the ICU were infected with drug-resistant strains of Acinetobacter species, $54.8 \%$ of the strains being XDR and $20.5 \%$ of the strains being MDR. The increased drug resistance seen in the ICU 
settings is probably because of the extensive use of broad-spectrum antibiotics for the critically ill patients. The mortality in the ICU settings was $30.14 \%$. All the 22 patients who expired were admitted in the ICU.

Risk factors like prolonged duration of stay in the hospital, intubation, mechanical ventilation, post-operative cases, and chronic underlying conditions such as diabetes mellitus, hypertension, ischemic heart disease, COPD, chronic liver disease, CKD, and malignancy were analyzed. Among these, intubation was the most common underlying predisposing factor present in $81 \%$ of the patients. Among the patients suffering from respiratory tract infections with Acinetobacter species, $86.9 \%$ were intubated during the course of the admission. All the 14 patients suffering from skin and soft tissue infections due to Acinetobacter species, were post-operative cases and the specimen was taken from the site of operation. Among the 5 patients suffering from urinary tract infection due to Acinetobacter species, 4 patients had urinary catheters inserted. Studies, conducted by Tripathi et al. [16] and Lone et al. [6] found that mechanical ventilation, admission to ICU, underlying chronic debilitating condition and a prolonged hospital stay were significant predisposing factors. The increased occurrence of infection by Acinetobacter species in patients with underlying chronic conditions reiterates the important opportunistic characteristic of Acinetobacter species, which affects compromised hosts. Prolonged hospital stay of more than a week also predisposes the patient to the hospital environment and stresses on the nosocomial character of the organism. $83 \%$ of the patients in our study had a prolonged hospital stay of more than a week. Acinetobacter strains have the ability to adhere to the surfaces by forming biofilm [18]. This is probably the reason why intubation and instrumentation like catheterization expose the person to infections caused by Acinetobacter species.

The occurrence of bacteremia due to Acinetobacter species in our study was $10 \%$. Out of the 10 patients, 3 were secondary to an indwelling catheter, 2 were secondary to VAP, and 2 were secondary to a urinary tract infection, while in 3 patients, the source was unknown. Prashanth et al. in their study found the incidence of bacteremia to be $16.27 \%$ with the most common source being respiratory tract infection (65\%) [5]. Intravascular catheters and respiratory tract infections are the most common sources of bacteremia by Acinetobacter species, while urinary tract infections and surgical wounds are the less common sources. These stress the importance of indwelling catheter care and infection control policies in preventing bloodstream infections due to Acinetobacter species. Some of the bacteremia's with source unknown could be secondary to undiagnosed vascular catheter infections or have an intestinal origin due to bacterial translocation. This is supported by the demonstration that digestive tract colonization by A. baumannii is more frequent and advanced in ICU patients $[19,20]$.

In our study, the mortality due to infection caused by Acinetobacter species was $22 \%$. The presence of risk factors in our study was statistically significant to mortality. Mechanical ventilation significantly increased the chances of mortality. Earlier studies reported a mortality rate of $23 \%$ in their study, with mechanical ventilation and infection with drug-resistant isolates as the risk factors associated with mortality [5]. Ventilator-associated pneumonia due to nonfermenting Gram-negative bacteria like Acinetobacter species is a known cause of increased mortality in hospitals [21]. Mortality and poor clinical outcomes are also due to high incidence of drug resistance in Acinetobacter species. This leaves the clinician with few treatment options and also the empiric therapy given is not effective.

Individually, among all the risk factors, mechanical ventilation was significantly associated with increase in drug resistance of the isolate. Furthermore, the presence of five or more risk factors is significantly associated with increase in drug resistance. Fukuta et al. and Surasarang et al. in their individual studies found mechanical ventilation, prolonged intensive unit care stay and prior antimicrobial use to be significant risk factors for acquisition of infection due to MDR Acinetobacter isolates [22,23]. Pertaining solely to the ICU settings, the risk factors that significantly lead to increase in drug resistance were found out to be mechanical ventilation, endotracheal intubation, post-operative cases and COPD and CKD. In addition to more severe health conditions of patients in ICU settings, cross-contamination, to a large extent, is associated with the high drug resistant rate, which has been demonstrated by numerous outbreaks of MDR isolates in critical centers [24,25]. Accordingly, to avoid the transmission of environmental organisms to patients from contaminated hands of medical staff and equipment, it is of great necessity and importance to reinforce the infection control strategies like hand washing and bundle care, especially in the ICU setting.

The Acinetobacter isolates causing infection in the ICU settings were significantly more resistant to antibiotics as compared to the isolates causing infection in the Ward settings $(\mathrm{p}<0.05)$. In our study, $57 \%$ of the Acinetobacter isolates which caused infection were carbapenemresistant. Intra-hospital and inter-hospital spread of MDR Acinetobacter species, especially carbapenem-resistant A. baumannii is becoming an issue of concern and leads to further spread of drug-resistant Acinetobacter strains, leaving no option apart from polymyxins for treatment [26]. Because of the same reasons, mortality was also found out to be significantly high in patients with infection with drug resistant Acinetobacter strains rather than susceptible Acinetobacter strains $(\mathrm{p}<0.05)$.

\section{CONCLUSION}

Acinetobacter species, especially A. baumannii is an important cause of severe nosocomial infections and hospital outbreaks; hence, this study of Acinetobacter species and their antibiotic susceptibility pattern is of immense significance. Although the resistance rates for these pathogens have been increasing steadily, the development of new drugs for these infections has not increased proportionately. Infections due to MDR Acinetobacter species increase the cost of treatment and severely affects clinical outcome and has very limited treatment options which include polymixins. The present scenario of escalating drug resistance in Acinetobacter species highlights the importance of uncompromising approach towards infection control, especially in the ICUs. An attempt to study the risk factors and the conditions that predispose a person to infections due to Acinetobacter species will help the treating physician to approach a patient and manage the infection along with the underlying condition appropriately.

\section{REFERENCES}

1. Peleg AY, Seifert H, Paterson DL. Acinetobacter baumannii: Emergence of a successful pathogen. Clin Microbiol Rev 2008;21(3):538-82.

2. Sinha N, Agarwal J, Srivastava S, Singh M. Analysis of carbapenemresistant Acinetobacter from a tertiary care setting in North India. Indian J Med Microbiol 2013;31(1):60-3.

3. Singla P, Sikka R, Deep A, Seema, Chaudhary U. Pattern of antimicrobial resistance in clinical isolates of Acinetobacter species at a tertiary level health care facility in Northern India. J Evol Med Dent Sci 2013;2(2):159-65.

4. García-Garmendia JL, Ortiz-Leyba C, Garnacho-Montero J, JiménezJiménez FJ, Pérez-Paredes C, Barrero-Almodóvar AE, et al. Risk factors for Acinetobacter baumannii nosocomial bacteremia in critically ill patients: A cohort study. Clin Infect Dis 2001;33(7):939-46.

5. Prashanth K, Badrinath S. Nosocomial infections due to Acinetobacter species: Clinical findings, risk and prognostic factors. Indian J Med Microbiol 2006;24(1):39-44

6. Lone R, Shah A, Kadri SM, Lone S, Faisal S. Nosocomial multidrug-resistant Acinetobacter infections-clinical findings, risk factors and demographic characteristics. Bangladesh J Med Microbiol 2009;3(1):34-8.

7. Cardoso T, Ribeiro O, Aragão IC, Costa-Pereira A, Sarmento AE. Additional risk factors for infection by multidrug-resistant pathogens in healthcare-associated infection: A large cohort study. BMC Infect Dis 2012;12:375.

8. American Thoracic Society; Infectious Diseases Society of America. Guidelines for the management of adults with hospital-acquired, ventilator-associated, and healthcare-associated pneumonia. Am J Respir Crit Care Med 2005;171(4):388-416. 
9. Clinical and Laboratory Standards Institute. Performance Standards for Antimicrobial Susceptibility Testing; Twenty-Third International Supplement. M100-S23. Wayne, PA: CLSI; 2013.

10. Choi JY, Kim CO, Park YS, Yoon HJ, Shin SY, Kim YK, et al. Comparison of efficacy of cefoperazone/sulbactam and imipenem/ cilastatin for treatment of Acinetobacter bacteremia. Yonsei Med J 2006;47(1):63-9.

11. Principe L, D’Arezzo S, Capone A, Petrosillo N, Visca P. In vitro activity of tigecycline in combination with various antimicrobials against multidrug resistant Acinetobacter baumannii. Ann Clin Microbiol Antimicrob 2009;8:18

12. Magiorakos AP, Srinivasan A, Carey RB, Carmeli Y, Falagas ME, Giske CG, et al. Multidrug-resistant, extensively drug-resistant and pandrug-resistant bacteria: An international expert proposal for interim standard definitions for acquired resistance. Clin Microbiol Infect 2012;18(3):268-81.

13. Vaze ND, Emery CL, Hamilton RJ, Brooks AD, Joshi SG. Patient demographics and characteristics of infection with carbapenemresistant Acinetobacter baumannii in a teaching hospital from the United States. Adv Infect Dis 2013;3(1):10-6.

14. Lee SO, Kim NJ, Choi SH, Hyong Kim T, Chung JW, Woo JH, et al. Risk factors for acquisition of imipenem-resistant Acinetobacter baumannii: A case-control study. Antimicrob Agents Chemother 2004;48(1):224-8.

15. Tilley PA, Roberts FJ. Bacteremia with Acinetobacter species: Risk factors and prognosis in different clinical settings. Clin Infect Dis 1994;18(6):896-900

16. Tripathi PC, Gajbhiye SR, Agrawal GN. Clinical and antimicrobial profile of Acinetobacter spp: An emerging nosocomial superbug. Adv Biomed Res 2014;3:13.

17. Cisneros JM, Rodríguez-Baño J. Nosocomial bacteremia due to Acinetobacter baumannii: Epidemiology, clinical features and treatment. Clin Microbiol Infect 2002;8(11):687-93.

18. Howard A, O'Donoghue M, Feeney A, Sleator RD. Acinetobacter baumannii: An emerging opportunistic pathogen. Virulence 2012;3(3):243-50

19. Corbella X, Pujol M, Ayats J, Sendra M, Ardanuy C, Domínguez MA, et al. Relevance of digestive tract colonization in the epidemiology of nosocomial infections due to multi resistant Acinetobacter baumannii. Clin Infect Dis 1996;23(2):329-34.

20. Timsit JF, Garrait V, Misset B, Goldstein FW, Renaud B, Carlet J. The digestive tract is a major site for Acinetobacter baumannii colonization in intensive care unit patients. J Infect Dis 1993;168(5):1336-7.

21. Falagas ME, Bliziotis IA, Siempos II. Attributable mortality of Acinetobacter baumannii infections in critically ill patients: A systematic review of matched cohort and case-control studies. Crit Care 2006;10(2):R48

22. Fukuta Y, Muder RR, Agha ME, Clarke LG, Wagener MM, Hensler AM, et al. Risk factors for acquisition of multidrug-resistant Acinetobacter baumannii among cancer patients. Am J Infect Control 2013;41(12):1249-52.

23. Surasarang K, Narksawat K, Danchaivijitr S, Siripanichgon K, Sujirarat D, Rongrungrueng Y, et al. Risk factors for multi-drug resistant Acinetobacter baumannii nosocomial infection. J Med Assoc Thai 2007;90(8):1633-9.

24. Anunnatsiri S, Tonsawan P. Risk factors and clinical outcomes of multidrug-resistant Acinetobacter baumannii bacteremia at a university hospital in Thailand. Southeast Asian J Trop Med Public Health 2011;42(3):693-703.

25. Liu WL, Liang HW, Lee MF, Lin HL, Lin YH, Chen CC, et al. The impact of inadequate terminal disinfection on an outbreak of imipenemresistant Acinetobacter baumannii in an intensive care unit. PLoS One 2014;9(9):e107975.

26. Pooja BM, Sweta RS, Sheela DK, Dileep VK. Characterization of carbapenem resistant Acinetobacter baumannii isolated in a tertiary care hospital: Epidemiology and treatment outcome. Int J Pharm Pharm Sci 2016;8(7):277-81. 